\author{
Ya. S. Budzhak ${ }^{1}$, T. Wacławski ${ }^{2}$
}

\title{
The Important Thermal Characteristics of Matter and Their Computations with the Use of the Gibbs Potentials
}

\author{
${ }^{1}$ State University Lvivska Polytechnica, Lviv-79013, Ukraine, \\ ${ }^{2}$ Cracow University of Technology, Cracow31-155, Poland, e-mail: tadeuszwaclawski00@ gmail.com
}

\begin{abstract}
In this paper, the important thermal characteristics of matter (they describe thermodynamic systems in a state of thermodynamic equilibrium) were calculated. There are the following important thermodynamic functions: the system internal energy $U$, the thermal function (or enthalpy) $H$, the free Helmholtz energy $F$, the thermodynamic potential (or Gibbs free energy) $G$, the Gibbs grand thermodynamic potential $\Omega$, the entropy $S$, the specific heat capacity $C_{V}$. These functions are explicit functions of system's parameters, they fulfil some mathematical relationships and possess some total differentials. These functions are calculated in this paper and their physical sense is given in the cited works.
\end{abstract}

Keywords: Gibbs potential, chemical potential, system's heat capacity, equation of neutrality.

Article acted received 10.06.2018; accepted for publication 15.06.2018.

\section{General statistical thermodynamics of thermal properties of matter}

In works [1-3] was shown that the thermodynamic properties of gaseous state are described by the statistical sum $Z_{N}$ for the classical ideal gas, or by the grand canonical sum $\Xi$ for the quantum gas.

Moreover, the statistical sum $\Xi$ of the quantum gas describes adequately the thermal properties of the ideal gas and the conducting crystals, as well as the radiation of bodies.

It was shown that the classical thermodynamic potentials of the ideal gas are given by the following general formulas:

$$
\begin{gathered}
U=N k T^{2} \frac{d \ln (Z(k T))}{d T}=\frac{3}{2} N k T \\
H=N k T\left[1+T \frac{d \ln Z(k T))}{d T}\right]=\frac{5}{2} N k T \\
F=-N k T\left[\ln \left(\frac{Z(k T)}{n}\right)+1\right]=N k T\left[\ln \left(\frac{n}{Z(k T)}\right)-1\right], \\
G=N k T \ln \left(\frac{Z(k T)}{n}\right) \\
\Omega=-N k T
\end{gathered}
$$

$$
\begin{gathered}
C_{V}=\left(\frac{d U}{d T}\right)_{V}, \\
P V=-\Omega=N k T,
\end{gathered}
$$

$$
n=\frac{N}{V}=-\left(\frac{d \Omega}{d \mu}\right)
$$


of the monatomic ideal gas and $Z(k T)=\left(\frac{2 \pi m k T}{h^{2}}\right)^{3 / 2}$, where $m$ is the mass of the gas particle. Physical sense of these potentials is given in the cited works.

The quantity $\ln (\Xi)$ determines the Gibbs grand thermodynamic potential $\Omega$. This potential describes all thermodynamic properties of the systems being considering and is given by the following algorithmic formula:

$$
\Omega=-k T \ln (\Xi)=-k T \sum_{i=0}^{i \rightarrow \infty} \ln \left(1+\exp \left(\frac{\mu-\varepsilon_{i}}{k T}\right)\right) .
$$

Applying now to the last formula the formal transition between summation and integration, we have:

$$
\Omega=-V \int_{0}^{\infty} \frac{G(\varepsilon)}{\left(\exp \left(\frac{\varepsilon-\mu}{k T}\right)+1\right)} d \varepsilon=-V \int_{0}^{\infty} G(\varepsilon) f_{0}(\varepsilon) d \varepsilon,
$$

where $f_{0}(\varepsilon)$ is the known Fermi-Dirac function, $G(\varepsilon)=\int_{0}^{\varepsilon} g(\varepsilon) d \varepsilon, \quad g(\varepsilon)$ is the density of the energy states (DOS) lying in allowed bands, $\varepsilon$ is the quantum particle energy - it depends on the particle quasimomentum $\dot{p}$. The function $\varepsilon(\dot{p})$ is called the dispersion relation. According to quantum mechanics, the energy dispersion relation $\varepsilon(\dot{p})$ is a periodic and even function of the vector $\stackrel{p}{p}$, the density of the energy states $g(\varepsilon)$ and the quantity $G(\varepsilon)$ are given by the

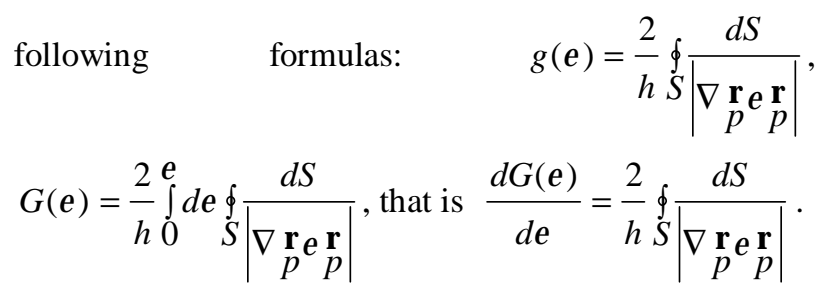

These formulas show that systems which may be described by the grand statistical sum (that is by the grand thermodynamic potential $\Omega$ ), strongly depend on the structure of the energy dispersion relation. Besides, in these systems, there may be degenerate or nondegenerate quantum gas particles.

The thermodynamic potentials (1-8) and their physical meaning are described in the cited works [2, 3]. These thermodynamic potentials (1-8) are expressed in terms of the Gibbs grand thermodynamic canonical potential and are given here by the following general algorithmic formulas:

$$
\begin{aligned}
& U_{n}=\Omega_{n}-\left(\frac{d \Omega_{n}}{d \mu}\right)_{T} \mu-\left(\frac{d \Omega_{n}}{d T}\right)_{\mu} T=N_{n} \cdot \frac{J(\varepsilon g(\varepsilon))}{J(g(\varepsilon))}, \\
& H_{n}=-\left(\frac{d \Omega_{n}}{d \mu}\right)_{\Gamma} \mu-\left(\frac{d \Omega_{n}}{d T}\right)_{\mu} T=N_{n} \cdot \frac{J\left(\frac{d}{d \varepsilon}(\varepsilon g(\varepsilon))\right)}{J(g(\varepsilon))}
\end{aligned}
$$

$$
\begin{gathered}
F_{n}=\Omega_{n}-\left(\frac{d \Omega_{n}}{d \mu}\right)_{T} \mu=N_{n}\left(\mu-\frac{J(G(\varepsilon))}{J(g(\varepsilon))}\right) \\
G_{n}=-\left(\frac{d \Omega_{n}}{d \mu}\right)_{T} \mu=N_{n} \mu \\
\Omega_{n}=-k T \ln \left(\Xi_{n}\right)=-N_{n} \frac{J(G(\varepsilon))}{J(g(\varepsilon))} \\
S_{n}=-\left(\frac{d \Omega_{n}}{d T}\right)_{\mu}=\frac{U_{n}-F_{n}}{T}=N_{n} k\left(\frac{d}{d \varepsilon}(\varepsilon G(\varepsilon))\right) \\
C_{V n}=\left(\frac{d(g(\varepsilon))}{d T}\right)_{V}, \\
P V=-\Omega_{n}=k T \ln \left(\Xi_{n}\right)=N_{n} \frac{J(G(\varepsilon))}{J(g(\varepsilon))}, \\
\frac{N_{n}}{V}=-\left(\frac{d \Omega_{n}}{d \mu}\right)=\int_{0}^{\infty} g(\varepsilon) f_{0}(\varepsilon) d \varepsilon=J(g(\varepsilon))=n_{n}
\end{gathered}
$$

In the above formulas, the calculated quantities of the electron or hole gas of the current carriers in a crystal are denoted by the subscript $n$. The integral is define as: $J(\varphi(\varepsilon))=\int_{0}^{\infty} \varphi(\varepsilon) f_{0}(\varepsilon) d \varepsilon$.

For non-degenerate current carriers, when $\frac{\mu}{k T}<-4$, the integral $J(\varphi(\varepsilon))$ has the following form:

$$
J(\varphi(\varepsilon))=\int_{0}^{\infty} \varphi(\varepsilon) \exp (-\varepsilon) d \varepsilon \cdot \exp \left(\frac{\mu}{k T}\right),
$$

For a strong degeneracy, when $\frac{\mu}{k T}>+4$, this integral may be approximated by the function of the form:

$$
J(\varphi(\varepsilon))=\int_{0}^{\mu_{0}} \varphi(\varepsilon) d \varepsilon\left[1+\frac{\pi^{2}}{6}(k T)^{2} \ln \left(\int_{0}^{\mu_{0}} \varphi(\varepsilon) d \varepsilon\right) \ln \left(\frac{\varphi\left(\mu_{0}\right)}{g\left(\mu_{0}\right)}\right)\right],
$$

where $\ln ^{\prime}\left(y\left(\mu_{0}\right)\right)=\frac{d \ln \left(y\left(\mu_{0}\right)\right)}{d \mu_{0}}$, and $\mu_{0}$ is the wellknown Fermi-Level.

Explication of a physical meaning of the degeneracy and non-degeneracy is given in the cited works.

Results of calculations of the thermal properties of the non-degenerate current carriers' gas (when formula (20) holds) fully agree with formulas (1-9) describing the thermal properties of the classical ideal gas.

When formulas (11-19) are compared with formulas (1-9), we must constantly keep in mind that the nondegenerate particles of the quantum gas fulfil the following relationships: 


$$
\begin{gathered}
n=\int_{0}^{\infty} g(\varepsilon) f_{0}(\varepsilon) d \varepsilon= \\
\int_{0}^{\infty} g(\varepsilon) e^{-\frac{\varepsilon}{k T}} d \varepsilon \cdot e^{\frac{\mu}{k T}}=Z(k T) e^{\frac{\mu}{k T}} ; Z(k T)=\int_{0}^{\infty} g(\varepsilon) e^{-\frac{\varepsilon}{k T}} d \varepsilon \\
\frac{n}{Z(k T)}=e^{\frac{\mu}{k T}} ; \ln \left(\frac{n}{Z(k T)}\right)=\frac{\mu}{k T}=\mu^{\bullet}
\end{gathered}
$$

These calculations show that the statistical sum of the non-degenerate quantum gas $Z(k T)$ is different from the statistical sum of the ideal gas $Z(k T)=\left(\frac{2 \pi m k T}{h^{2}}\right)^{3 / 2}$, where $m$ is the ideal gas' particle mass. Therefore, the quantum gas having this density is similar to the classical ideal gas, and its thermal properties are fully described by formulas (1-9).

It can be seen from this analysis that the grand thermodynamic potential $\Omega$ (formula (10)) describes adequately the thermodynamic functions of the quantum gas and thermodynamic functions of the classical ideal gas. And these gases are not identical each other. It is associated with the fact the statistical sum of the ideal gas $Z(k T)=\left(\frac{2 \pi m k T}{h^{2}}\right)^{3 / 2}$, and the statistical sum of the ideal quantum gas $Z(k T)=\int_{0}^{\infty} g(\varepsilon) e^{-\frac{\varepsilon}{k T}} d \varepsilon$. This quantum statistical sum depends on the dispersion relation $\varepsilon(\dot{p})$ of the quantum particles.

When the reduced chemical potential $\mu^{\bullet}=\frac{\mu}{k T} \geq+4$, the quantum gas may be in a degenerate state. It is easy to show that in this case the particles' density of degenerate gas $n=\frac{N}{V}$, according to formulas (19) and (21) is given by:

$$
n=\frac{N}{V}=\int_{0}^{\mu_{0}} g(\varepsilon) d \varepsilon=G\left(\mu_{0}\right)
$$

We shall use the classical Sommerfeld approximation to evaluate the integral in formula (19) to show the relations between the Fermi level $\mu_{0}$ and the chemical potential $\mu$ of the Fermi-particles. Thus, we have:

$$
n=\frac{N}{V}=\int_{0}^{\infty} g(\varepsilon) f_{0}(\varepsilon) d \varepsilon=\int_{0}^{\mu} g(\varepsilon) d \varepsilon+\frac{\pi^{2}}{6}(k T)^{2} \frac{d g(\mu)}{d \mu} \cong \int_{0}^{\mu_{0}} g(\varepsilon) d \varepsilon+\left(\mu-\mu_{0}\right) g\left(\mu_{0}\right)+\frac{\pi^{2}}{6}(k T)^{2} \frac{d g\left(\mu_{0}\right)}{d \mu_{0}}
$$

From this approximation and taking into consideration equality (23), it follows the equation:

$$
\left(\mu-\mu_{0}\right) g\left(\mu_{0}\right)+\frac{\pi^{2}}{6}(k T)^{2} \frac{d g\left(\mu_{0}\right)}{d \mu_{0}}=0,
$$

This equation is called the equation of neutrality of a degenerate Fermi-gas. This equation provides a possibility to determinate the chemical potential $\mu$ of the degenerate Fermi-particles and to find the relations between this chemical potential and the Fermi level $\mu_{0}$. An elementary solution of this equation has the following form:

$$
\mu=\mu_{0}\left[1-\frac{\pi^{2}}{6} \frac{(k T)^{2}}{\mu_{0} g\left(\mu_{0}\right)} \frac{d g\left(\mu_{0}\right)}{d \mu_{0}}\right],
$$

From equation (23), it can be found the Fermi level $G\left(\mu_{0}\right)=n$. It is clear from this relationship that the Fermi level $\mu_{0}$ depends on the Fermi particles' density and their dispersion relation $\varepsilon(p)$ via the function $G\left(\mu_{0}\right)$.
In the case of the ideal Fermi gas for a parabolic dispersion relation $\varepsilon(\stackrel{p}{p})$, we have:

$$
G\left(\mu_{0}\right)=\frac{8}{3 \sqrt{\pi}}\left(\frac{2 \pi m}{h^{2}}\right)^{3 / 2} \mu_{0}^{3 / 2}
$$

Thus, $\mu_{0}=\left(\frac{3 n}{8 \pi}\right)^{2 / 3} \frac{h^{2}}{2 m}$.

It can be seen that in the case of isotropic crystals with the isotropic parabolic dispersion relations $\varepsilon(p)$ (when the the current carriers are strongly degenerate), the chemical potential $\mu$ and the Fermi level have the following form:

$$
\begin{gathered}
\mu(T)=\mu_{0} \cdot\left(1-\frac{\pi^{2}}{12} \cdot\left(\frac{k T}{\mu_{0}}\right)\right), \\
\mu_{0}=\left(\frac{3 n}{8 \pi}\right)^{\frac{2}{3}} \frac{h^{2}}{2 m},
\end{gathered}
$$




\section{An elementary model of a crystal. The statistical sum of a crystal.}

The crystal of interest to us is a thermodynamic system. This system is composed of two thermodynamic subsystems of particles. One system - this is a gas of charge carriers to be considered as a grand canonical ensemble of particles. The other - this is a set of structure particles. These particles vibrate around their fixed equilibrium in the crystal lattice.

The statistical sum $\Xi_{k}$ of this model of the crystal is equal to the product of two statistical sums: the statistical sum of the charge carriers' gas in the crystal $\Xi_{n}$ and the statistical sum of the structure particles' gas $\Xi_{N}$. Thus, we have $\Xi_{k}=\Xi_{n} \Xi_{N}$.

In the cited works it was shown that the thermal properties (potentials) of large thermodynamic system are described in terms of the logarithm of a statistical sum. But the logarithm $\ln \Xi_{k}=\ln \Xi_{n}+\ln \Xi_{N}$. Physically, it means that the thermal potentials of the crystal should be additive added the thermal potentials of the crystal lattice and the thermal potentials of the charge carriers' gas.

According to statistical physics, in the conducting crystals, the thermal properties of the current carriers' gas may be described by the Gibbs grand potential:

$$
\Omega_{n}=-k T \ln \left(\Xi_{n}\right)=-V \int_{0}^{\infty} \frac{G(\varepsilon)}{\left(\exp \left(\frac{\varepsilon-\mu}{k T}\right)+1\right)} d \varepsilon=-V \int_{0}^{\infty} G(\varepsilon) f_{0}(\varepsilon) d \varepsilon
$$

and therefore, we get formulas (11-19) providing algorithms for the computations of these thermal potentials.

In the case of the crystal lattice, according to the Debye approximation, the Gibbs grand potential may be described by the following formula:

$$
\Omega_{N}=3 N k T\left[3 \ln \left(1-\exp \left(-\frac{\theta}{T}\right)\right)-D\left(\frac{\theta}{T}\right)\right],
$$

Commonly, this formula is called the Debye's interpolation formula. In this formula $\theta$ is the Debye temperature which depends on the nature of the crystal,

$D(Z)=\frac{3}{Z^{3}} \int_{0}^{Z} \frac{x^{3} d x}{(\exp (x)-1)}$ is the Debye function, $N$ is the density of the crystal's structure particles.

Having the thermodynamic potential $\Omega_{N}$, the known algorithmic formulas permit the calculation of all thermodynamic properties of the crystal lattice, when the chemical potential of phonons is equal to zero, $(\mu=0)$. Thus, we have:

$$
U_{N}=\Omega_{N}-\left(\frac{d \Omega N}{d T}\right) T=3 N k T D\left(\frac{\theta}{T}\right),
$$

$$
C_{V N}=3 N k\left[4 D\left(\frac{\theta}{T}\right)-\frac{3}{\left(\exp \left(\frac{\theta}{T}\right)-1\right)}\right],
$$

$$
(P V)_{N}=-\Omega_{N}=3 N k T\left[D\left(\frac{\theta}{T}\right)-3 \ln \left(1-\exp \left(-\frac{\theta}{T}\right)\right)\right] \text {, }
$$

The general thermal properties of the crystal should be additive added the thermal properties of the crystal lattice and the thermal properties of the Fermi gas' particles. The thermal properties of this Fermi gas are 
described by the general formulas (11)-(19). Because of this, the general thermodynamic properties of the crystal are given by the following relationships:

$$
\begin{gathered}
U_{k}=U_{N}+U_{n}=U_{N}\left[1+\frac{U_{n}}{U_{N}}\right], \\
H_{k}=H_{N}+H_{n}=H_{N}\left[1+\frac{H_{n}}{H_{N}}\right], \\
F_{k}=F_{N}+F_{n}=F_{N}\left[1+\frac{F_{n}}{F_{N}}\right], \\
G_{k}=0, \quad\left[\begin{array}{c}
\Omega_{n} \\
\Omega_{k}=\Omega_{N}+\Omega_{n}
\end{array}\right], \\
C_{v k}=C_{V N}+C_{V n}=C_{V N}\left[1+\frac{C_{V n}}{C_{V N}}\right], \\
(P V)_{k}=-\Omega_{D}-\Omega_{e}=-\Omega\left(1+\frac{\Omega_{n}}{\Omega_{N}}\right],
\end{gathered}
$$

In the above formulas, the subscripts $k, N, n$ are for the thermodynamic properties of the crystal, the crystal lattice, and the charge carriers' gas, respectively.

Practical computations show that formulas (I)-(VII) adequately describe the thermal functions of simple crystals. Theoretical analysis of these formulas also confirms this fact.

\section{Conclusions}

In conclusions, the Gibbs grand thermodynamic potential $\Omega$ adequately describes the thermal functions of simple crystals. This potential can be used to justify and determine to the Gibbs canonical distribution, the Gibbs grand canonical distribution, the Maxwellian and Maxwell-Boltzmann distributions. This same potential can successfully be used to determine the thermal properties of matter, and it describes (as was shown in the works $[2,3])$, laws of the thermal radiation of bodies.

Budjak Ja.S. - Professor, Doctor of Physical and Mathematical Sciences;

Wactawski T. - PhD adjunct.

[1] Ya. S. Budzhak, Physic and Chemistry of Solid States 18(1), 7 (2017).

[2] Ya. S. Budzhak, V. Chaban, Energy and kinetic properties of semiconductor crystals (ProstirM, Lviv, 2017).

[3] Ya.S. Budzhak, L.O. Vasilechko, Foundations of statistical theory of thermal and kinetic properties of semiconductor crystals (Liga-Pres, Lviv, 2010).

\author{
Я.С. Буджак ${ }^{1}$, Т. Вацлавски
}

\title{
Важливі теплові характеристики речовин та їх розрахунки за допомогою потенціалів Гіббса
}

\footnotetext{
${ }^{1}$ Наиіональний університет «Львівська політехніка»,в. С.Бандери, 12, м. Львів, Україна, 79013, е-таil: jabudjak@ukr.net ${ }^{2}$ Краківська політехніка, в. Варшавска, 24, м. Краків, Польща, 31-155, e-mail: tadeuszwaclawski00@ gmail.com
}

В даній роботі розраховані важливі теплові характеристики речовин, які описують термодинамічні системи в стані термодинамічної рівноваги. До важливих термодинамічних функцій, як матеріальних властивостей системи, відносяться: внутрішня енергія системи $U$, теплова функція, або ентальпія $H$, вільна енергія Гельмгольца $F$, термодинамічний потенціал (або вільна енергія Гіббса) $G$, великий термодинамічний потенціал Гіббса $\Omega$, ентропія $S$, питома теплоємність $C_{V}$. Ці функції є явними функціями певних параметрів системи, відповідають певним математичним відношенням і володіють певними повними диференціалами. Вони розраховані в приведеній роботі, а їх фізичний зміст описаний в цитованій літературі.

Ключові слова: потенціал Гіббса, хімічний потенціал, теплоємність системи, рівняння нейтральності. 